\title{
Changing the frequency and spectra of chromosomal aberrations in Korean patients with acute leukemia in a tertiary care hospital
}

\author{
Je-Hyun Park ${ }^{1,2, \#}$, Min-Gu Kang ${ }^{3, \#}$, Hye-Ran Kim, ${ }^{4, \#}$, Young-Eun Lee ${ }^{2}$, Jun Hyung Lee ${ }^{1}$, \\ Hyun-Jung Choi ${ }^{1}$, Jong-Hee Shin ${ }^{1}$, Myung-Geun Shin ${ }^{1,2}$ \\ ${ }^{1}$ Department of Laboratory Medicine, Chonnam National University Medical School and Chonnam National University Hwasun \\ Hospital, ${ }^{2}$ Brain Korea 21 Plus program, Chonnam National University Medical School and Chonnam National University Hwasun \\ Hospital, Hwasun, ${ }^{3}$ Department of Laboratory Medicine, Gwang Yang Sarang General Hospital, Gwangyang, ${ }^{4}$ College of Korean \\ Medicine, Dongshin University, Naju, Korea
}

\begin{abstract}
p-ISSN 2287-979X / e-ISSN 2288-0011 https://doi.org/10.5045/br.2020.2020255 Blood Res 2020;55:225-245.
\end{abstract}

Received on October 7, 2020 Accepted on October 30, 2020

${ }^{\#}$ These authors contributed equally to this study.

\author{
*This study was supported by a grant from \\ National Research Foundation of Korea \\ (NRF) grant funded by the Korean \\ government (MSIT) (No.2019R1F1A10 \\ 59859) and the Ministry of Education \\ (No.2018R1D1A1B07040984). \\ Correspondence to \\ Myung-Geun Shin, M.D., Ph.D. \\ Department of Laboratory Medicine, \\ Chonnam National University Medical \\ School and Chonnam National University \\ Hwasun Hospital, 322 Seoyang-ro, \\ Hwasun 58128, Korea \\ E-mail: mgshin@chonnam.ac.kr \\ (C) 2020 Korean Society of Hematology
}

\begin{abstract}
Background
Chromosomal analysis is essential for the diagnosis and risk stratification of all leukemia patients. Not surprisingly, racial differences in chromosomal aberrations (CA) in hematological malignancies could be found, and CA incidence in leukemia might change over time, possibly due to environmental and lifestyle changes. Thus, we compared the frequency and range of CA in patients with acute leukemia $(A L)$ during two time periods (2006-2009 vs. 2010-2015) and compared them with other prior studies.
\end{abstract}

\section{Methods}

We enrolled 717 patients with AL during a six-year period (2010-2015). We compared the results to those of our earlier study (2006-2009) [1]. Conventional cytogenetics, a multiplex reverse transcriptase (RT)-PCR system, and fluorescence in situ hybridization were employed to assess bone marrow specimens or peripheral blood at the diagnostic stage in $\mathrm{AL}$ patients to detect $\mathrm{CA}$.

Results

The incidence of CA changed in the leukemia subgroups during the two time periods. Notably, the most frequent CA of childhood acute myeloid leukemia (AML) was $P M L / R A R A$, and was followed by $R U N X 1 / R U N X 1 T 1$ in the current study. In contrast, the most common CA was $R U N X 1 / R U N X 1 T 1$ in a previous study [1] and was followed by $P M L / R A R A$. In this study, the most frequent CA of the mixed phenotype AL was $B C R / A B L 1$, which was followed by $K M T 2 A / M L L T 3$. In a previous report, [1] the most frequent CA was $B C R / A B L 1$, which was followed by $K M T 2 A / E L L$.

\section{Conclusion}

The distribution of CA in Korean AL patients changed over time in a single institute. This change might be due to environmental and lifestyle changes.

Key Words Acute leukemia, Chromosomal aberrations, Frequency, Spectra

\section{INTRODUCTION}

Chromosomal aberrations (CA) in hematologic malignancies can be commonly examined using conventional cytogenetics, fluorescence in situ hybridization (FISH), and multiplex reverse transcriptase polymerase chain reaction (multiplex RT-PCR). A commercially available multiplex RT-PCR system has been introduced to detect 28 fusion genes in patients with acute leukemia (AL) [1].

CA develops in almost $56 \%$ of adult de novo acute myeloid leukemia (AML) and slightly more often in childhood cases [2-4]. Racial differences in hematological malignancies between Asian and Western countries have been reported [5, 6]. The prevalence of CA in AL might change over time, possibly due to changes in various risk factors, such as environmental pollutants and lifestyle, as mentioned in a previous study $[7,8]$. Therefore, we compared the frequency and 
spectra of CA in patients with AL during two time periods (2006-2009 vs. 2010-2015) in a single institute and with other previous reports.

\section{MATERIALS AND METHODS}

\section{Patients}

In total, 717 patients with AL were enrolled during a 6-year period (2010-2015). This study included 125 children (median age, $8 \mathrm{yr}$; range, 0-18 yr) and 592 adults (median age, $57 \mathrm{yr}$; range, $19-88 \mathrm{yr})$. A total of $66 \%$ (83/125) of the children were male and $34 \%(42 / 125)$ were female; $58 \%$ (341/592) of the adults were male and $42 \%$ (251/592) were female; $73 \%$ (521/717) were diagnosed with primary $(84 \%)$ or secondary (16\%) AML (40 children, 481 adults), 26\% (187/717) were diagnosed with acute lymphoid leukemia (ALL) (80 children, 107 adults), and 1\% (9/717) were diagnosed with mixed phenotype acute leukemia (MPAL) (Supplementary Table 1). Clinical and laboratory data, such as sex, age, and the results of multiplex RT-PCR, FISH, and conventional cytogenetics were obtained from electronic medical records (EMR). This study was approved by the institutional review board (CNUHH No. 2009-35). Informed consent was obtained from all participating patients.

Multiplex RT-PCR system

In total, $89 \%(637 / 717)$ of $\mathrm{AL}$ patients were analyzed using the multiplex RT-PCR system. Briefly, RNA was extracted from the peripheral blood (PB) or bone marrow (BM) using a commercial kit (RNAqueous kit; Ambion, Austin, TX, USA), in accordance with the manufacturer's protocol. The multiplex RT-PCR test kit (DNA Technology, Aarhus, Denmark) was used to detect 28 of the most common leukemic fusion genes and more than 80 splice variants (Supplementary Fig. 1).

\section{Fluorescence in situ hybridization (FISH)}

FISH was performed in appropriate $\mathrm{BM}$ or $\mathrm{PB}$ specimens available from 609 patients, in addition to the conventional cytogenetic method. It was performed using an appropriate probe (Abbott Molecular/Vysis, Des Plaines, IL, USA), in accordance with the manufacturer's instructions.

\section{Conventional cytogenetics}

Conventional cytogenetic analysis was performed at the initial diagnosis phase using short-term cultures of BM cells. At least 20 metaphases were analyzed in each case, and the clonal CA was described according to the International System for Cytogenetic Nomenclature 2009 and 2013 (Supplementary Fig. 2).

Table 1. Frequency and spectra of chromosomal aberrations according to the type of all acute leukemia patients using the multiplex RT-PCR system.

\begin{tabular}{|c|c|c|c|c|c|}
\hline $\begin{array}{l}\text { Chromosomal aberration using } \\
\text { multiplex RT-PCR system }\end{array}$ & Fusion transcript & AML & ALL & MPAL & Total \\
\hline $\mathrm{t}(15 ; 17)(\mathrm{q} 24 ; \mathrm{q} 21)$ & $P M L / R A R A$ & 67 & & & 67 \\
\hline $\mathrm{t}(9 ; 22)(\mathrm{q} 34 ; \mathrm{q} 11)$ & $B C R / A B L 1$ & 9 & 34 & 4 & 47 \\
\hline$t(8 ; 21)(q 22 ; q 22)$ & $R U N X 1 / R U N X 1 T 1$ & 34 & & & 34 \\
\hline \multicolumn{6}{|l|}{$11 q 23$} \\
\hline $\mathrm{t}(4 ; 11)(\mathrm{q} 21 ; \mathrm{q} 23)$ & KMT2A/AFF1 & & 3 & & 3 \\
\hline $\mathrm{t}(6 ; 11)(\mathrm{q} 27 ; \mathrm{q} 23)$ & $K M T 2 A / A F D N$ & 5 & & & 5 \\
\hline $\mathrm{t}(11 ; 19)(\mathrm{q} 23 ; \mathrm{p} 13.3)$ & KMT2A/ELL & 2 & & & 2 \\
\hline $\mathrm{t}(9 ; 11)(\mathrm{p} 22 ; \mathrm{q} 23)$ & KMT2A/MLLT3 & 8 & & 1 & 9 \\
\hline $\mathrm{t}(10 ; 11)(\mathrm{p} 12 ; \mathrm{q} 23)$ & KMT2A/MLLT1O & 5 & 1 & & 6 \\
\hline $\mathrm{t}(11 ; 19)(\mathrm{q} 23 ; \mathrm{p} 13.3)$ & $K M T 2 A / M L L T 1$ & & 1 & & 1 \\
\hline $\mathrm{t}(12 ; 21)(\mathrm{p} 13 ; \mathrm{q} 22)$ & ETV6/RUNX1 & & 15 & & 15 \\
\hline $\mathrm{t}(1 ; 19)(q 23 ; p 13)$ & TCF3/PBX1 & & 5 & & 5 \\
\hline $\operatorname{inv}(16)(p 13 ; q 22)$ & $C B F B / M Y H 11$ & 23 & & & 23 \\
\hline $\mathrm{t}(9 ; 9)(\mathrm{q} 34 ; \mathrm{q} 34)$ & SET/NUP214 & 1 & 1 & & 2 \\
\hline $\operatorname{del}(1 \mathrm{p} 32)$ & STIL/TAL1 & & 4 & & 4 \\
\hline $\mathrm{t}(16 ; 21)(\mathrm{p} 11 ; \mathrm{q} 22)$ & FUS/ERG & 3 & & & 3 \\
\hline $\mathrm{t}(6 ; 9)(p 23 ; q 34)$ & DEK/NUP214 & 2 & & & 2 \\
\hline $\mathrm{N}$ of positive cases & & 159 & 64 & 5 & 228 \\
\hline Total cases & & 521 & 187 & 9 & 717 \\
\hline NT & & 61 & 19 & 0 & 80 \\
\hline Cases excluding ' $N T^{\prime}$ & & 460 & 168 & 9 & 637 \\
\hline Positive cases excluding 'NT' (\%) & & 35 & 38 & 56 & 36 \\
\hline
\end{tabular}

Abbreviations: ALL, acute lymphoid leukemia; AML, acute myeloid leukemia; MPAL, mixed-phenotype acute leukemia; NT, not tested. 


\section{RESULTS}

\section{Detection of CA using a multiplex RT-PCR system}

Sixteen CA types were detected in 36\% (228/637) of the patients using a multiplex RT-PCR system (Supplementary Table 2). The multiplex RT-PCR system detected fusion transcripts in 35\% (159/460) of AML patients, 38\% (64/168) of ALL patients, and 56\% (5/9) of MPAL patients (Table 1).

\section{Detection of CA using conventional cytogenetics, including FISH}

In total, conventional cytogenetic methods were performed in 97\% (506/521) of the AML patients, 94\% (175/187) of the ALL patients, and $100 \%$ (9/9) of the MPAL patients. Among these, 93\% of AML, 87\% of ALL, and 100\% of MPAL were successfully analyzed. Successful analyses were achieved in 92\% (634/690) of the patients. Among the successfully analyzed cases, 59\% (372/634) had detectable CA and $41 \%(262 / 634)$ were considered cytogenetically normal (Table 2).

Thirteen types of fusion transcripts, excluding extra-aberrations, were detected in 29\% (202/690) of patients using conventional cytogenetics. Meanwhile, the extra-aberration indicated numerical abnormalities or structural rearrangements, excluding 28 fusion transcripts, in the multiplex RT-PCR system. The most frequent CA was $\mathrm{t}(15 ; 17)(\mathrm{q} 24 ; \mathrm{q} 21)$ in $9.7 \%(67 / 690)$, followed by $\mathrm{t}(9 ; 22)$ (q34;q11) in 5.9\% (41/690), $\mathrm{t}(8 ; 21)(\mathrm{q} 22 ; \mathrm{q} 22)$ in $5.2 \%(36 / 690)$, $\operatorname{inv}(16)(\mathrm{p} 13 ; \mathrm{q} 22)$ in $3.2 \%(22 / 690)$, and $K M T 2 A$ gene rearrangements in $2.8 \%(19 / 690)$ of patients (Table 2, Supplementary Table 3). Within a group of 59\% (23/39) of childhood AML patients, the most common CA was $\mathrm{t}(15 ; 17)(\mathrm{q} 24 ; \mathrm{q} 21)(\mathrm{N}=8)$, followed by $\mathrm{t}(8 ; 21)(\mathrm{q} 22 ; \mathrm{q} 22)(\mathrm{N}=6)$ (Supplementary Table 4). Within a group of $25 \%(19 / 75)$ of childhood ALL patients, the CA was $\mathrm{t}(12 ; 21)(\mathrm{p} 13 ; \mathrm{q} 22)$ in 12 cases, followed by $\mathrm{t}(9 ; 22)(\mathrm{q} 34 ; \mathrm{q} 11)$ in five cases, and one case each of $t(10 ; 11)(p 12 ; q 23)$ and $t(11 ; 19)(q 23 ; p 13.3)$ (Supplementary Table 4). However, extra-aberrations were the majority in childhood ALL patients. Within a group of $27 \%(128 / 467)$ of adult AML patients, the most common CA was $\mathrm{t}(15 ; 17)(\mathrm{q} 21 ; \mathrm{q} 22)(\mathrm{N}=59)$, followed by $\mathrm{t}(8 ; 21)(\mathrm{q} 22 ; \mathrm{q} 22)$ $(\mathrm{N}=30)$ (Supplementary Table 4 ). Within a group of $27 \%$ $(27 / 100)$ of adult ALL patients, the common CA was $\mathrm{t}(9 ; 22)(\mathrm{q} 34 ; \mathrm{q} 11)(\mathrm{N}=25)$. Meanwhile, the majority of adult patients showed a normal karyotype (Supplementary Table 4).

Table 2. Frequency and spectra of chromosomal aberrations according to the type of acute leukemia and detection method in all patients using conventional cytogenetics, including FISH.

\begin{tabular}{|c|c|c|c|c|c|c|c|c|}
\hline \multirow{2}{*}{$\begin{array}{l}\text { Chromosomal aberration by } \\
\text { conventional cytogenetics }\end{array}$} & \multirow{2}{*}{ Fusion transcript } & \multicolumn{2}{|c|}{ AML } & \multicolumn{2}{|c|}{ ALL } & \multicolumn{2}{|c|}{ MPAL } & \multirow{2}{*}{ Total } \\
\hline & & K & $\mathrm{F}$ & K & $\mathrm{F}$ & K & $\mathrm{F}$ & \\
\hline $\mathrm{t}(15 ; 17)(\mathrm{q} 24 ; \mathrm{q} 21)$ & $P M L / R A R A$ & 54 & 13 & & & & & 67 \\
\hline $\mathrm{t}(9 ; 22)(\mathrm{q} 34 ; \mathrm{q} 11)$ & $B C R / A B L 1$ & 3 & 4 & 22 & 8 & 4 & & 41 \\
\hline $\mathrm{t}(8 ; 21)(\mathrm{q} 22 ; \mathrm{q} 22)$ & $R U N \times 1 / R U N X 1 T 1$ & 36 & & & & & & 36 \\
\hline \multicolumn{9}{|l|}{$11 q 23$} \\
\hline $\mathrm{t}(4 ; 11)(\mathrm{q} 21 ; \mathrm{q} 23)$ & KMT2A/AFF1 & & & 1 & & & & 1 \\
\hline $\mathrm{t}(6 ; 11)(\mathrm{q} 27 ; \mathrm{q} 23)$ & $K M T 2 A / A F D N$ & 5 & & & & & & 5 \\
\hline $\mathrm{t}(11 ; 19)(q 23 ; p 13.3)$ & $K M T 2 A / E L L$ & & & & & & & 0 \\
\hline $\mathrm{t}(9 ; 11)(\mathrm{p} 22 ; \mathrm{q} 23)$ & KMT2A/MLLT3 & 6 & & & & 1 & & 7 \\
\hline $\mathrm{t}(10 ; 11)(\mathrm{p} 12 ; \mathrm{q} 23)$ & KMT2A/MLLT1O & 4 & & 1 & & & & 5 \\
\hline $\mathrm{t}(11 ; 19)(\mathrm{q} 23 ; \mathrm{p} 13.3)$ & KMT2A/MLLT1 & & & 1 & & & & 1 \\
\hline $\mathrm{t}(12 ; 21)(\mathrm{p} 13 ; \mathrm{q} 22)$ & $E T V 6 / R U N X 1$ & & & 5 & 7 & & & 12 \\
\hline $\mathrm{t}(1 ; 19)(q 23 ; p 13)$ & TCF3/PBX1 & & & 1 & & & & 1 \\
\hline $\operatorname{inv}(16)(p 13 ; q 22)$ & $C B F B / M Y H 11$ & 21 & 1 & & & & & 22 \\
\hline $\mathrm{t}(9 ; 9)(\mathrm{q} 34 ; \mathrm{q} 34)$ & SET/NUP214 & & & & & & & 0 \\
\hline $\operatorname{del}(1 p 32)$ & STIL/TAL1 & & & & & & & 0 \\
\hline $\mathrm{t}(16 ; 21)(\mathrm{p} 11 ; \mathrm{q} 22)$ & FUS/ERG & 2 & & & & & & 2 \\
\hline $\mathrm{t}(6 ; 9)(\mathrm{p} 23 ; \mathrm{q} 34)$ & DEK/NUP214 & 2 & & & & & & 2 \\
\hline Extra-aberration ${ }^{\text {a) }}$ & & 113 & 5 & 43 & 7 & 2 & & 170 \\
\hline \multicolumn{2}{|c|}{ Positive cases excluding extra-aberration } & \multicolumn{2}{|c|}{151} & \multicolumn{2}{|c|}{46} & \multicolumn{2}{|c|}{5} & 202 \\
\hline \multicolumn{2}{|l|}{ Positive cases } & \multicolumn{2}{|c|}{269} & \multicolumn{2}{|c|}{96} & \multicolumn{2}{|c|}{7} & 372 \\
\hline \multicolumn{2}{|l|}{ Total cases } & \multicolumn{2}{|c|}{521} & \multicolumn{2}{|c|}{187} & \multicolumn{2}{|c|}{9} & 717 \\
\hline \multicolumn{2}{|l|}{ Cases of 'NT' } & \multicolumn{2}{|c|}{15} & \multicolumn{2}{|c|}{12} & \multicolumn{2}{|c|}{0} & 27 \\
\hline \multicolumn{2}{|l|}{ Cases excluding ' $N T^{\prime}$} & \multicolumn{2}{|c|}{506} & \multicolumn{2}{|c|}{175} & \multicolumn{2}{|c|}{9} & 690 \\
\hline \multicolumn{2}{|c|}{ Positive cases excluding 'extra-aberration' \& 'NT' (\%) } & \multicolumn{2}{|c|}{30} & \multicolumn{2}{|c|}{26} & \multicolumn{2}{|c|}{56} & 29 \\
\hline Positive cases excluding ' & & & & & & & & 54 \\
\hline
\end{tabular}

${ }^{a)}$ Extra-aberrations are the numerical abnormalities or structural rearrangements, excluding 28 fusion transcripts of the multiplex RT-PCR system.

Abbreviations: F, fluorescence in situ hybridization; K, karyotype; NT, not tested. 
Frequency and spectra of CA according to childhood and adult groups using multiplex RT-PCR

A total of $46 \%$ percent $(54 / 117)$ of the childhood group subjects exhibited a positive result using the multiplex RT-PCR system. Within a group of $65 \%$ (24/37) of childhood AML patients, the common CA included eight $P M L / R A R A$, followed by five $R U N X 1 / R U N X 1 T 1$, five $K M T 2 A$ gene rearrangements (three $K M T 2 A / M L L T 3$ and two $K M T 2 A / M L L T 10$ ), three $C B F B / M Y H 11$, and one of each of $B C R / A B L 1$, $D E K / N U P 214$, and FUS/ERG. In a group of $37 \%(28 / 75)$ of childhood ALL patients, the following fusion scripts were detected: $15 E T V 6 / R U N X 1,5 B C R / A B L 1$, four $K M T 2 A$ gene rearrangements (two $K M T 2 A / A F F 1$, one each of $K M T 2 A$ / $M L L T 1$ and KMT2A/MLLT19), three STIL/TAL1, and one TCF/PBX1. A total of $33 \%(174 / 520)$ of adult ALL patients had a positive result. Within a group of $32 \%(135 / 423)$ of adult AML patients, the common CA included $P M L / R A R A$ (N=59), followed by RUNX1/RUNX1T1 ( $\mathrm{N}=29), C B F B / M Y H 11$ $(\mathrm{N}=20), K M T 2 A$ gene rearrangements $(\mathrm{N}=15)[K M T 2 A / A F D N$ $(\mathrm{N}=5), \quad K M T 2 A / M L L T 3(\mathrm{~N}=5), \quad K M T 2 A / M L L T 10(\mathrm{~N}=3)$, $K M T 2 A / E L L(\mathrm{~N}=2)], B C R / A B L 1(\mathrm{~N}=8), F U S / E R G(\mathrm{~N}=2)$, $D E K / N U P 214(\mathrm{~N}=1)$, and SET/NUP214 $(\mathrm{N}=1)$. The common CA in $39 \%(36 / 93)$ of adult ALL patients included $B C R / A B L 1$ $(\mathrm{N}=29)$, followed by $T C F 3 / P B X 1(\mathrm{~N}=4), K M T 2 A / A F F 1(\mathrm{~N}=1)$, SET/NUP214 $(\mathrm{N}=1)$, and STIL/TAL1 $(\mathrm{N}=1)$ (Supplementary Table 5).
Frequency and spectra of CA according to AML and ALL patient groups using multiplex RT-PCR

In a group of $35 \%(159 / 460)$ of AML patients, the following fusion transcripts were detected: $67 P M L / R A R A, 34 R U N X 1 /$ RUNX1T1, 23 CBFB/MYH11, 20 KMT2A rearrangements, nine $B C R / A B L 1$, three FUS/ERG, two DEK/NUP214, and one $S E T / N U P 214$. In a group of $38 \%(64 / 168)$ of ALL patients, fusion transcripts included $34 B C R / A B L 1,15 E T V 6 / R U N X 1$, five $T C F 3 / P B X 1$, five $K M T 2 A$ gene rearrangements (three $K M T 2 A / A F F 1$, one of each of KMT2A/MLLT1 and KMT2A/ MLLT19), four STIL/TAL1, and one SET/NUP214. In a group of $56 \%(5 / 9)$ of MPAL patients, the following fusion transcripts were detected: four $B C R / A B L 1$ and one $K M T 2 A$ / MLLT3 (Table 1).

\section{Results from multiplex RT-PCR system and conventional} cytogenetics

There was an agreement between the multiplex RT-PCR system and conventional cytogenetics in 71\% (394/553) of patients. No CA was detected using either method in $41 \%$ (228/553) of patients. Thirteen types of fusion transcripts were detected using a multiplex RT-PCR system in 30\% $(166 / 553)$ of the patients, which was in agreement with conventional cytogenetics. Conventional cytogenetics disclosed additional structural rearrangements and/or numerical abnormalities in $11 \%(61 / 553)$ of the patients with specific aberrations, which were demonstrated using both methods. The results of the multiplex RT-PCR system did not correspond with the conventional cytogenetics in 29\% (159/553)

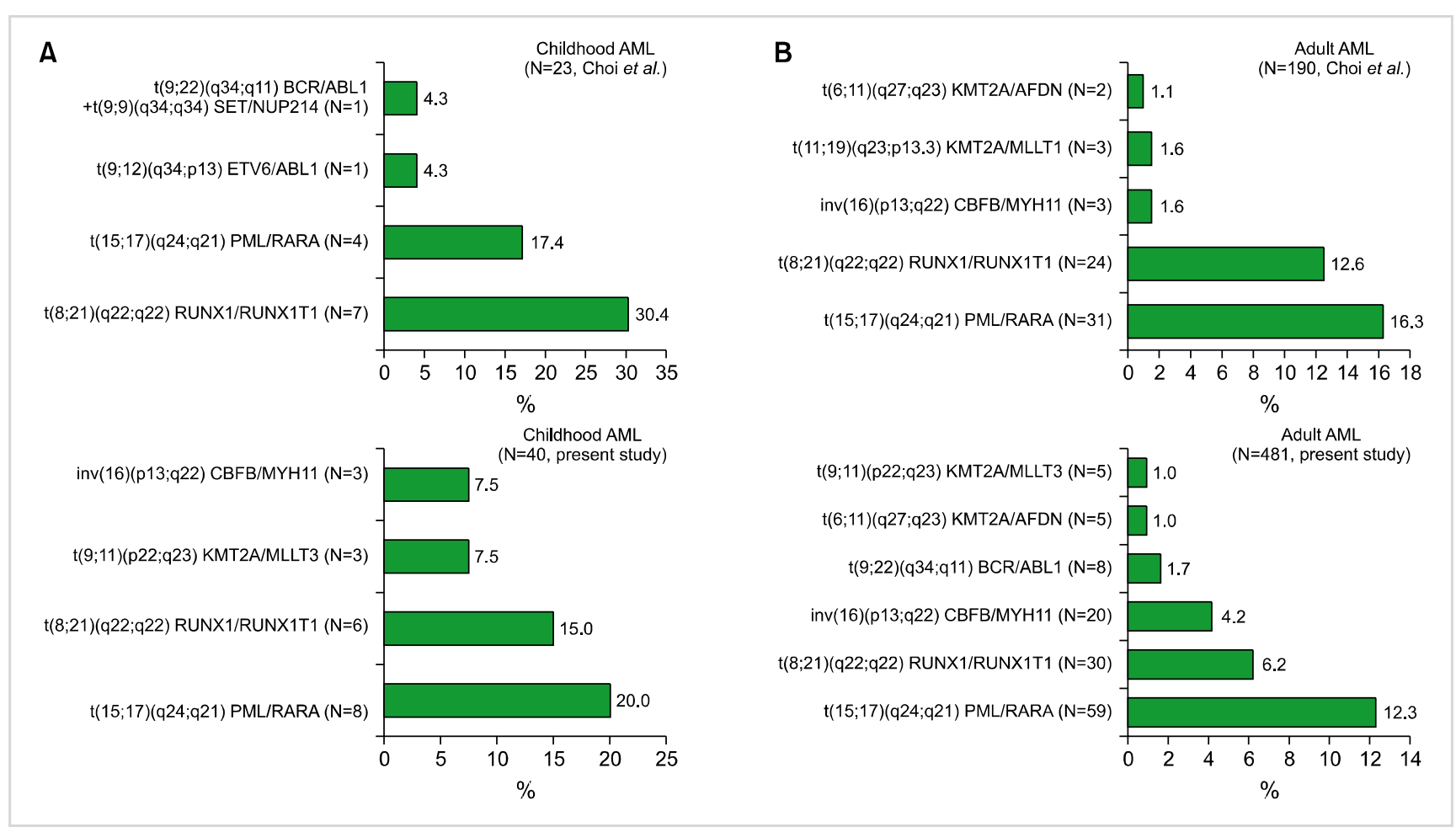

Fig. 1. The prevalence of major chromosomal aberrations, which were detected using conventional cytogenetics, including FISH and multiplex RT-PCR, were compared to the previous study [1] and described according to leukemia type and age. (A) Childhood AML, (B) adult AML. 
of the patients. Cytogenetically cryptic translocations solely detected using multiplex RT-PCR system were present in $5 \%(28 / 553)$ of the patients with a normal karyotype, numerical abnormalities, or structural rearrangements based on conventional cytogenetics. Detailed comparison data between the multiplex RT-PCR system and conventional cytogenetics are summarized in Supplementary Table 6. A total of $36 \%(131 / 359)$ of the patients with a negative result based on the multiplex RT-PCR system had CA, according to conventional cytogenetics results (Supplementary Table 6-8).

Considering both PCR methods and conventional cytogenetics, the most frequent CA of childhood and adult AML was $P M L / R A R A$, which was followed by RUNX1/RUNX1T1. The most frequent CA of childhood and adult ALL was ETV6/RUNX1 and BCR/ABL1, respectively (Supplementary Table 9, 10, Fig. 1, 2). The most frequent CA of the mixed phenotype AL was $B C R / A B L 1$, which was followed by KMT2A/MLLT3 (Table 1, 2).

Important CA that were not covered by the multiplex RT-PCR system

In the current study, 98 types of CA were determined in 717 patients with hematological malignancies. The multiplex RT-PCR system for screening 28 fusion transcripts identified only 16 types among them. Eighty-two types of leukemic fusion could not be found using multiplex RT-PCR, excluding numerical abnormalities. Three important CAs, such as $\mathrm{i}(17)(\mathrm{q} 10), \mathrm{t}(3 ; 3)(\mathrm{q} 21 ; \mathrm{q} 26.2)$, and $\mathrm{t}(8 ; 14)(\mathrm{q} 24 ; \mathrm{q} 32)$, were found using conventional cytogenetics (Supplementary Table 7, 8, Supplementary Fig. 2).

\section{DISCUSSION}

Considering the many fusion genes and breakpoint variants currently determined, more than 50 separate PCR reactions are required to screen an $\mathrm{AL}$ patient with a standard operation [9]. We adopted a commercially accessible multiplex-RT-PCR system (DNA Technology) to identify 28 common fusion genes and over 80 splice variants based on previous research [9]. Data from Denmark using this system, which was employed on specimens from 143 patients with a median age of 63 years (range, 0-85 yr; 132 adults, 11 children), revealed that CA was identified in only $15 \%$ (21/143) of the patients [10]. In contrast, this study showed a higher CA frequency of $36 \%(228 / 637)$, using the same multiplex RT-PCR system: 35\% (159/460) of AML patients, $38 \%(64 / 168)$ of ALL patients, and $56 \%$ (5/9) of MPAL patients. The rate of occurrence and spread of leukemic fusion genes in $\mathrm{AL}$ also differed from earlier European data [10]. CA occurred in 65\% (24/37) of childhood AML cases, whereas it only occurred in about $40 \%(24 / 60)$ of childhood AML cases based on the same multiplex RT-PCR system from Austria [11]. The frequency of leukemic fusion genes in our ALL patients $(38 \%, 64 / 168)$ was analogous to an Italian dataset utilizing the same multiplex RT-PCR system: $37 \%$ (28/75) and 39\% (36/93) of childhood and adult ALL patients, respectively. In the Italian study, 39\% of 170 ALL patients carried leukemic fusion genes: $39 \%$ and $40 \%$ of the childhood and adult ALL patients, respectively [12].

The most common CA in AML patients was the $\mathrm{t}(15 ; 17)$

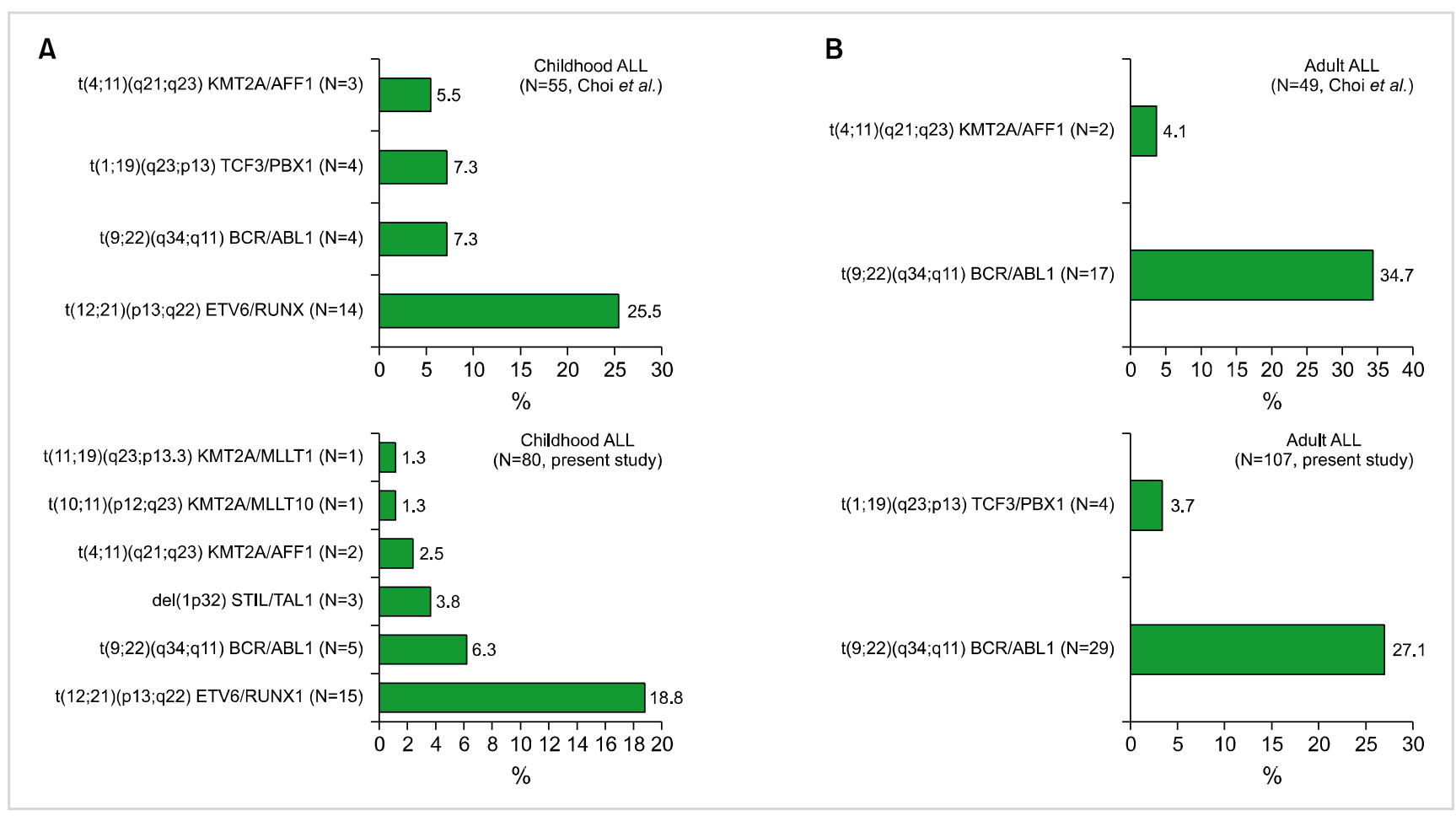

Fig. 2. The prevalence of major chromosomal aberrations, which were detected using conventional cytogenetics, including FISH and multiplex RT-PCR, was compared to that of the previous study [1] and described according to leukemia type and age. (A) Childhood ALL, (B) adult ALL. 
abnormality $(15 \%, 67 / 460)$. The occurrence rate was higher compared to data from other institutes of Korea (6.7\%), Caucasian (6.5, 10\%), Australian (12\%), Japanese (11\%), and Singapore-Chinese (11\%), but it was analogous to the Chinese data $(14.3 \%)[5,6,13]$. This discrepancy may be due to the sample size and likely to ethnic differences. The geographic diversity of CA in leukemia needs further study and a better understanding of the genetic and environmental factors associated with leukemia. The frequency and range of CA in ALL were similar between this study and previous studies $[6,14]$. A balanced translocation of the $B C R / A B L 1$ fusion gene in ALL patients was the most common abnormality $(20 \%, 34 / 168)$ in this study. The occurrence of the $B C R / A B L 1$ fusion transcript was lower than in the Southwest Oncology Group data (26\%) [15], while it was higher than those in Indian (6\%) and Taiwanese data $(8 \%)$ [16, 17]. The most common CA in childhood ALL patients was the $E T V 6 / R U N X 1$ fusion transcript, which was followed by $B C R / A B L 1, K M T 2 A$ gene rearrangements, STIL/TAL, and $T C F 3 / P B X 1$. In contrast, the $B C R / A B L 1$ fusion transcript was the most common CA in adult ALL patients, which was followed by TCF3/PBX1, KMT2A/AFF1, SET/NUP214, and STIL/TAL1.

The discrepancy of $29 \%$ (159/553) between the multiplex RT-PCR system and conventional cytogenetics was mainly due to numerical and submicroscopic anomalies. The multiplex RT-PCR system used to screen 28 fusion transcripts identified 16 types in the group of 98 types of CA determined in 717 patients with leukemia. The concordance rate of $71 \%$ (394/553) between the multiplex RT-PCR system and conventional karyotyping was analogous to an earlier study (70\%) [1].

Choi et al. [1] reported 20 fusion transcripts containing KMT2A/EPS15 (MLL1/AF-1p), ETV6/ABL1 (TEL/ABL), RUNX1/MDS1 (AML1/MDS1), ZBTB16/RARA (PLZF/RARA), and $E T V 6 / M N 1$ (TEL/MN1) using a multiplex RT-PCR system. However, 16 fusion transcripts containing $D E K /$ NUP214 were determined using the multiplex RT-PCR system in this study, and the above-mentioned 5 fusion transcripts were not identified: KMT2A/EPS15 (MLL1/AF-1p), ETV6/ABL1 (TEL/ABL), RUNX1/MDS1 (AML1/MDS1), ZBTB16/ RARA (PLZF/RARA), and ETV6/MN1 (TEL/MN1).

In addition, the range or spectra of leukemic fusion genes in $\mathrm{AL}$ differed from a prior report in Korea (Fig. 1, 2) [1]. In a prior study, Choi et al. examined 325 leukemia patients during a 4-year period (2006-2009). Eighty-one children (median age $8 \mathrm{yr}$, range $0-18 \mathrm{yr}$ ) and 244 adults (median age $56 \mathrm{yr}$, range $19-86 \mathrm{yr}$ ) were included in their study. Of the 81 children, 35 (43\%) were male and 46 (57\%) were female; of the 244 adults, $123(50 \%)$ were male. A total of $213(66 \%)$ patients were diagnosed with de novo (92\%) or secondary (8\%) AML (23 children, 190 adults); 104 (32\%) patients had ALL (55 children, 49 adults), and 8 had mixed phenotype acute leukemia (MPAL). Accordingly, these findings were somewhat heterogeneous and different from those of the present study, as mentioned in the Patients' section of the materials in this study.
In addition, the most prevalent CA in childhood AML patients was $P M L / R A R A$, and was followed by $R U N X 1 /$ RUNX1T1 in this study (Supplementary Table 9), whereas the most prevalent CA was $R U N X 1 / R U N X 1 T 1$, which was followed by $P M L / R A R A$ in a previous report [1]. In addition, taking into account MPAL patients, the most prevalent CA was $B C R / A B L 1$, followed by $K M T 2 A / M L L T 3$, in this study (Supplementary Table 9, 10), while the most common CA was $B C R / A B L 1$, followed by $K M T 2 A / E L L$ in a previous report [1].

Interestingly, there were cases with significant $C A$, such as $\mathrm{i}(17)(\mathrm{q} 10), \mathrm{t}(3 ; 3)(\mathrm{q} 21 ; \mathrm{q} 26.2)$, and $\mathrm{t}(8 ; 14)(\mathrm{q} 24 ; \mathrm{q} 32)$, that were not identified using the commercial multiplex RT-PCR system (Supplementary Table 8, Supplementary Fig. 2). These are very meaningful and typical CA in hematological malignancies, such as AL. $t(8 ; 14)(q 24 ; q 32)$ is a typical CA in patients with ALL (Burkitt type), and the $\mathrm{t}(3 ; 3)(\mathrm{q} 21 ; \mathrm{q} 26.2)$ is a newly adopted recurrent CA in AML patients, in accordance with the 2008 WHO classification [1]. In addition, $\mathrm{i}(17)(\mathrm{q} 10)$ is repeatedly shown in the blast crisis of Philadelphiapositive CML patients. As a solitary CA, i(17q) has also been demonstrated in primary fibrosis, hypereosinophilic syndrome, and rare cases of myelodysplastic syndrome (MDS) and myeloproliferative neoplasm (MPN) that result in acute non-lymphocytic leukemia. In accordance with several studies, MDS/MPN, which contains the $\mathrm{i}(17 \mathrm{q})$ as a single CA, is regarded as a distinctive entity with a frequency of $0.4-$ $1.57 \%$ of MDS, or $1 \%$ of all myeloid cases, which features a male predominance, significant anemia, hyposegmented neutrophils, increased micromegakaryocytes, and an unfavorable prognosis $[18,19]$. Thus, it is worth considering $\mathrm{CA}$ as an additional screening panel of leukemic fusion genes for improving the molecular detection system.

In conclusion, this study disclosed the frequency and range of CA in Korean AL patients, which differed from those of previous published reports. This change might be due to environmental and lifestyle changes $[7,8]$. Our data were collected from a single center in Korea; thus, the findings may not be generalizable to other institutions. In addition, the limited number of patients might preclude definitive conclusions on the AL changes in Korea. Further studies to accumulate more evidence are needed to determine the change in spectra or range of AL. Nonetheless, this study might provide important information for improving the molecular detection system in Korean AL patients.

\section{ACKNOWLEDGMENTS}

MGS designed the study. JHP and YEL enrolled the patients, collected clinical laboratory data, and performed laboratory measurements. HRK initially set up a multiplex RT-PCR detection system. JHP wrote the preliminary manuscript. MGS, JHP, and MGK analyzed the data. MGK, MGS, and HRK revised the manuscript. JHL, HJC, and JHS interpreted the data and provided valuable comments and recommendations. All the authors have revised and accepted 
the final version of the manuscript.

\section{Authors' Disclosures of Potential Conflicts of Interest}

No potential conflicts of interest relevant to this article were reported.

\section{REFERENCES}

1. Choi HJ, Kim HR, Shin MG, et al. Spectra of chromosomal aberrations in 325 leukemia patients and implications for the development of new molecular detection systems. J Korean Med Sci 2011;26:886-92.

2. Bacher U, Kern W, Schnittger S, Hiddemann W, Schoch C, Haferlach T. Further correlations of morphology according to FAB and WHO classification to cytogenetics in de novo acute myeloid leukemia: a study on 2,235 patients. Ann Hematol 2005;84:785-91.

3. Klaus M, Haferlach T, Schnittger S, Kern W, Hiddemann W, Schoch C. Cytogenetic profile in de novo acute myeloid leukemia with $\mathrm{FAB}$ subtypes M0, M1, and M2: a study based on 652 cases analyzed with morphology, cytogenetics, and fluorescence in situ hybridization. Cancer Genet Cytogenet 2004;155:47-56.

4. Betts DR, Ammann RA, Hirt A, et al. The prognostic significance of cytogenetic aberrations in childhood acute myeloid leukaemia. A study of the Swiss Paediatric Oncology Group (SPOG). Eur J Haematol 2007;78:468-76.

5. Nakase K, Bradstock K, Sartor M, et al. Geographic heterogeneity of cellular characteristics of acute myeloid leukemia: a comparative study of Australian and Japanese adult cases. Leukemia 2000; 14:163-8.

6. Cheng Y, Wang Y, Wang H, et al. Cytogenetic profile of de novo acute myeloid leukemia: a study based on 1432 patients in a single institution of China. Leukemia 2009;23:1801-6.

7. Xie Y, Davies SM, Xiang Y, Robison LL, Ross JA. Trends in leukemia incidence and survival in the United States (1973-1998). Cancer 2003;97:2229-35.

8. Irigaray P, Newby JA, Clapp R, et al. Lifestyle-related factors and environmental agents causing cancer: an overview. Biomed Pharmacother 2007;61:640-58.
9. Pallisgaard N, Hokland P, Riishøj DC, Pedersen B, Jørgensen P. Multiplex reverse transcription-polymerase chain reaction for simultaneous screening of 29 translocations and chromosomal aberrations in acute leukemia. Blood 1998;92:574-88.

10. Hutchings Hoffmann $M$, Wirenfeldt Klausen $T$, Hasle $H$, Schmiegelow K, Brondum-Nielsen K, Johnsen HE. Multiplex reverse transcription polymerase chain reaction screening in acute myeloid leukemia detects cytogenetically unrevealed abnormalities of prognostic significance. Haematologica 2005;90: 984-6.

11. Strehl S, König M, Mann G, Haas OA. Multiplex reverse transcriptase-polymerase chain reaction screening in childhood acute myeloblastic leukemia. Blood 2001;97:805-8.

12. Elia L, Mancini M, Moleti L, et al. A multiplex reverse transcriptase-polymerase chain reaction strategy for the diagnostic molecular screening of chimeric genes: a clinical evaluation on 170 patients with acute lymphoblastic leukemia. Haematologica 2003;88:275-9.

13. Jung J, Cho BS, Kim HJ, et al. Reclassification of acute myeloid leukemia according to the 2016 WHO classification. Ann Lab Med 2019;39:311-6

14. Pui CH. Acute lymphoblastic leukemia in children. Curr Opin Oncol 2000;12:3-12.

15. Pullarkat V, Slovak ML, Kopecky KJ, Forman SJ, Appelbaum FR. Impact of cytogenetics on the outcome of adult acute lymphoblastic leukemia: results of Southwest Oncology Group 9400 study. Blood 2008;111:2563-72.

16. Amare P, Gladstone B, Varghese C, Pai S, Advani S. Clinical significance of cytogenetic findings at diagnosis and in remission in childhood and adult acute lymphoblastic leukemia: experience from India. Cancer Genet Cytogenet 1999;110:44-53.

17. Chang HH, Lu MY, Jou ST, Lin KH, Tien HF, Lin DT. Cytogenetics in childhood acute lymphoblastic leukemia in Taiwan: a singleinstitutional experience. Pediatr Hematol Oncol 2006;23: 495-506.

18. Pinheiro RF, Chauffaille Mde L, Silva MR. Isochromosome 17q in MDS: a marker of a distinct entity. Cancer Genet Cytogenet 2006; 166:189-90.

19. Kim MH, Hwang HY, Jeong SH, et al. Detection of p53 mutant and isochromosome 17q in myelodysplastic syndromes and leukemias. Korean J Clin Pathol 2000;20:349-53. 